(C) [2008] IEEE. Reprinted, with permission, from [Guangyuan Yang, Yong Zhang, Gang Lei, K. R. Shao, Youguang Guo, Jianguo Zhu and J. D. Laver,A Novel Superposition RBF Collocation Method to Solve Moving Conductor Eddy Current Problems, Magnetics, IEEE Transactions on Volume 45, Issue: 10 Oct 2009]. This material is posted here with permission of the IEEE. Such ermission of the IEEE does not in any way imply IEEE endorsement of any of the University of Technology, Sydney's products or services. Internal or personal use of this material is permitted.

However, permission to reprint/republish this material for advertising or promotional purposes or for creating new collective works for resale or redistribution must be obtained from the IEEE by writing to pubs-permissions@ieee.org. By choosing to view this document, you agree to all provisions of the copyright laws protecting it 


\section{A Novel Superposition RBF Collocation Method to Solve Moving Conductor Eddy Current Problems}

Guangyuan Yang, Yong Zhang, Gang Lei, K.R. Shao

Huazhong University of Science and Technology,

Wuhan, 430074, P.R. China

Youguang Guo, Jianguo Zhu

University of Technology, Sydney, NSW, Australia

\section{J.D. Lavers}

University of Toronto, Toronto, Ont. M5S 3G4, Canada 
Abstract -- This paper presents a novel radial basis function (RBF) collocation method to solve the moving conductor eddy current problem. The magnetic field is considered an addition of two fields generated respectively by the excitation current and the eddy current according to the source superposition principle. The corresponding governing equations are decoupled and solved with the RBF. Moving coordinate systems in which the separate fields are computed are also constructed to avoid the model reconfiguration caused by the motion. Electromagnetic field equations are analyzed with kinetic equations and circuit equations together to simulate the motion process. A practical engineering problem is computed to verify the method. 


\section{INTRODUCTION}

Until now, the computation of eddy current magnetic field with moving conductor has attracted much attention in engineering applications [1]-[2]. Since the position of moving conductor in the solving domain is always changing, traditional mesh-based methods, for instance, the finite element method (FEM), meet great difficulty in reforming the mesh when the motion process needs to be analyzed.

As a newly developed meshless method, the RBF collocation method introduces a set of nodes distributed in solving domain instead of traditional elements to form primary functions and the partial differential equations are transformed into linear matrix equations and solved [3]. Until now, it has been applied to compute steady and transient state electromagnetic fields [4]-[6].

According to the properties of RBF and the source superposition principle, this paper presents a novel algorithm to solve moving 
conductor eddy current problem and analyze the motion. The magnetic field is considered to be an addition of two fields generated by the excitation current and the eddy current respectively. The governing equations including two unknown fields are decoupled with RBF and solved by time-domain iteration. Moving coordinate systems in which separate fields are calculated are also constructed to avoid the model reconfiguration during the motion. Kinetic equations and circuit equations are solved on the basis of the magnetic fields to simulate the motion process. To verify this method, we computed the eddy current problem in a moving electromagnetic switch and analyzed its motion.

\section{MOVING CONDUCTOR EDDY CURRENT PROBLEM}

Consider a homogeneous and isotropic electromagnetic system in domain $\Omega \subset R^{n}$ with a boundary $\partial \Omega$ in which a non-magnetic 
conductor is moving with a speed $V$. The equations for the electromagnetic field are:

$$
\begin{array}{cc}
\nabla \times \boldsymbol{H}=\boldsymbol{J}_{e}+\boldsymbol{J}_{s} \quad, \quad \boldsymbol{J}_{e}=\sigma(\boldsymbol{E}+\boldsymbol{V} \times \boldsymbol{B}) \\
\nabla \times \boldsymbol{E}=-\frac{\partial \boldsymbol{B}}{\partial t} \quad, \quad \boldsymbol{B}=\mu \boldsymbol{H}
\end{array}
$$

where $\underset{s}{\text { Ind }_{s}}$ aregexcitation current and eddy current respectively. Assuming that $\sigma$ and $\mu$ are constant, with Lorentz gauge, the equivalent form of (1) in conductor area vector potential is: by usigg magnetic

$$
\nabla^{2} \boldsymbol{A}+\sigma \mu\left(\frac{\partial \boldsymbol{A}}{\partial t}-\boldsymbol{V} \times \nabla \times \boldsymbol{A}\right)=\mu \boldsymbol{J}_{s}
$$

This is a typical convective-diffusion equation. Since $J_{e}$ exists only in $\Omega_{e}$, we get the governing equations as follows: 


$$
\left\{\begin{array}{cc}
\sigma \mu \frac{\partial \boldsymbol{A}}{\partial t}-L(\boldsymbol{A})=\mu \boldsymbol{J}_{s} & \text { in } \Omega_{e} \\
\nabla^{2} \boldsymbol{A}=\mu \boldsymbol{J}_{s} & \text { in } \Omega-\Omega_{e} \\
B(\boldsymbol{A})=0 & \text { on } \partial \Omega
\end{array}\right.
$$

where the operator $L(.)^{\text {means }}\left(\sigma \mu \boldsymbol{V} \times \nabla \times-\nabla^{2}\right)$ and $B($.$) is a$ boundary operator.

\section{SUPERPOSITION RBF COLLOCATION METHOD}

With $N_{I}+N_{B}=N$ collocation nodes respectively in $\Omega$ and on $\partial \Omega$, the magnetic vector potential $A$ could be written as [3]:

$$
\boldsymbol{A}(\boldsymbol{x}, t)=\sum_{j=1}^{N} a_{j}(t) \varphi\left(\left\|\boldsymbol{x}-\boldsymbol{x}_{j}\right\|, c\right)=\boldsymbol{\varphi}^{T}(\boldsymbol{x}) \boldsymbol{a}(t)
$$

where $\boldsymbol{x}$ is the coordinate of nodes in the space, $\varphi\left(\left\|\boldsymbol{x}-\boldsymbol{x}_{j}\right\|, c\right)$ is the RBF centered at node $x_{j}$ and $\varphi(x)$ is the vector form, 
$\left\|x-x_{j}\right\|$ is the Euclidean distance, $a(t)$ is the unknown coefficient vector to be determined, $c$ is a shape parameter. And the RBF collocation form of (3) at node $\boldsymbol{x}_{i}$ is:

$$
\begin{array}{lc}
\sigma \mu \varphi^{T}\left(\boldsymbol{x}_{i}\right) \frac{\partial \boldsymbol{a}}{\partial t}-L\left(\boldsymbol{\varphi}^{T}\left(\boldsymbol{x}_{i}\right)\right) \boldsymbol{a}=\mu \boldsymbol{J}_{s}\left(\boldsymbol{x}_{i}, t\right) & \boldsymbol{x}_{i} \in \Omega_{e} \\
\left(\nabla^{2} \boldsymbol{\varphi}^{T}\left(\boldsymbol{x}_{i}\right)\right) \boldsymbol{a}=\mu \boldsymbol{J}_{s}\left(\boldsymbol{x}_{i}, t\right) & \boldsymbol{x}_{i} \in \Omega-\Omega_{e} \\
B\left(\boldsymbol{\varphi}^{T}\left(\boldsymbol{x}_{i}\right)\right) \boldsymbol{a}=0 & \boldsymbol{x}_{i} \in \partial \Omega
\end{array}
$$

Crank-Nicolson time matching scheme is applied to deal with the time differential and construct iteration to solve (5) as:

$$
\begin{array}{lc}
\left(\frac{\sigma \mu}{\Delta t} \boldsymbol{\varphi}^{T}\left(\boldsymbol{x}_{i}\right)-\frac{1}{2} L\left(\boldsymbol{\varphi}^{T}\left(\boldsymbol{x}_{i}\right)\right)\right) \boldsymbol{a}^{k+1}=\left(\frac{\sigma \mu}{\Delta t} \boldsymbol{\varphi}^{T}\left(\boldsymbol{x}_{i}\right)\right. & \\
\left.+\frac{1}{2} L\left(\boldsymbol{\varphi}^{T}\left(\boldsymbol{x}_{i}\right)\right)\right) \boldsymbol{a}^{k}+\mu \boldsymbol{J}_{s}^{k+1}\left(\boldsymbol{x}_{i}\right) & \boldsymbol{x}_{i} \in \Omega_{e} \\
\left(\nabla^{2} \boldsymbol{\varphi}^{T}\left(\boldsymbol{x}_{i}\right)\right) \boldsymbol{a}=\mu \boldsymbol{J}_{s}\left(\boldsymbol{x}_{i}, t\right) & \boldsymbol{x}_{i} \in \Omega-\Omega_{e} \\
B\left(\boldsymbol{\varphi}^{T}\left(\boldsymbol{x}_{i}\right)\right) \boldsymbol{a}^{k+1}=0 & \boldsymbol{x}_{i} \in \partial \Omega
\end{array}
$$


where $\nabla \times \varphi^{T}, L\left(\varphi^{T}\right)$ and $B\left(\varphi^{T}\right)$ mean doing operating to the elements in the vector $\varphi, \Delta t$ is the time interval.

Equation (6) shows the governing equations at nodes in different areas and on the boundary. To exactly figure the shapes of the conductor and the excitation current areas, sufficient nodes should be set in each part of the domain. However, when the conductor lies very close to the excitation area, the nodes in the gap between them satisfy different equations from those in the current-existing areas. Since the gap is too small to cover many nodes, it is difficult to exactly approximate the high gradient magnetic filed in it. Besides, if we consider the nodes moveless, the shape of the moving conductor can not be figured during the motion and the governing equations at nodes near the conductor boundary will change abruptly when the conductor covers or leaves them. Otherwise, if the nodes are assumed to move with the conductor, the RBF matrix will become time-variant to break the iteration. 
To solve these problems, according to the superposition principle, the magnetic field is considered to be an addition of two fields generated by $\boldsymbol{J}_{s}$ and $\boldsymbol{J}_{e}$ respectively as:

$$
\boldsymbol{A}(\boldsymbol{x}, t)=\boldsymbol{A}_{s}(\boldsymbol{x}, t)+\boldsymbol{A}_{e}(\boldsymbol{x}, t)=\boldsymbol{\varphi}_{s}^{T}(\boldsymbol{x}) \boldsymbol{a}_{s}(t)+\boldsymbol{\varphi}_{e}^{T}(\boldsymbol{x}) \boldsymbol{a}_{e}(t)
$$

The separate fields $\boldsymbol{A}_{s}$ and $\boldsymbol{A}_{e}$ are expressed with RBFs formed in different coordinate systems. Both $\varphi_{s}$ and $\varphi_{e}$ are formed on nodes located in the global solving domain and they could be different RBFs or structured by nodes at different positions. With this method, the gap between the conductor and the excitation area is eliminated and the motion of the conductor could be expressed by the relative movement between coordinate systems with fixed RBF nodes to avoid the model reconfiguration during iteration.

Consider $\boldsymbol{x}_{s}$ and $\boldsymbol{x}_{e}$ to be the coordinates of nodes of $\boldsymbol{\varphi}_{s}$ and $\boldsymbol{\varphi}_{e}$ in their own system and $\boldsymbol{x}_{s}^{\prime}$ and $\boldsymbol{x}_{\boldsymbol{e}}^{\prime}$ to be in the other's. $A_{s}$ satisfies the Poisson equation everywhere in $\Omega$. So we get: 


$$
\begin{array}{ll}
\left.\nabla^{2} \boldsymbol{\varphi}_{s}^{T}\left(\boldsymbol{x}_{s i}\right)\right) \boldsymbol{a}_{s}^{k+1}=\mu \boldsymbol{J}_{s}^{k+1}\left(\boldsymbol{x}_{s i}\right) & \boldsymbol{x}_{s i} \in \Omega \\
B\left(\boldsymbol{\varphi}_{s}^{T}\left(\boldsymbol{x}_{s i}\right)\right) \boldsymbol{a}_{s}^{k+1}=0 & \boldsymbol{x}_{s i} \in \partial \Omega
\end{array}
$$

With a known $\boldsymbol{J}_{s}$, the coefficient $\boldsymbol{a}_{s}^{k+1}$, which would be used to compute $a_{e}^{k+1}$ later, could be obtained. Substituting (7) and (8a) in to (6) and noticing that $\boldsymbol{F}_{e}$ xists only in $\Omega_{e}$ e get:

$$
\begin{array}{cc}
\left(\frac{\sigma \mu}{\Delta t} \boldsymbol{\varphi}_{e}^{T}\left(\boldsymbol{x}_{e i}\right)-\frac{1}{2} L\left(\boldsymbol{\varphi}_{e}^{T}\left(\boldsymbol{x}_{e i}\right)\right)\right) \boldsymbol{a}_{e}^{k+1}=\left(\frac{\sigma \mu}{\Delta t} \boldsymbol{\varphi}_{e}^{T}\left(\boldsymbol{x}_{e i}\right)+\frac{1}{2} L\left(\boldsymbol{\varphi}_{e}^{T}\left(\boldsymbol{x}_{e i}\right)\right)\right) \boldsymbol{a}_{e}^{k} \\
-\frac{\sigma \mu}{\Delta t}\left(\boldsymbol{A}_{s}^{k+1}\left(\boldsymbol{x}_{e i}^{\prime}\right)-\boldsymbol{A}_{s}^{k}\left(\boldsymbol{x}_{e i}^{\prime}\right)\right)+\sigma \mu \boldsymbol{V} \times \nabla \times \boldsymbol{A}_{s}^{k+1}\left(\boldsymbol{x}_{e i}^{\prime}\right) \quad \boldsymbol{x}_{e i} \in \Omega_{e} \\
\left.\nabla^{2} \boldsymbol{\varphi}_{e}^{T}\left(\boldsymbol{x}_{e i}\right)\right) \boldsymbol{a}_{e}^{k+1}=0 \\
B\left(\boldsymbol{\varphi}_{e}^{T}\left(\boldsymbol{x}_{e i}\right)\right) \boldsymbol{a}_{e}^{k+1}=0 & \boldsymbol{x}_{e i} \in \partial \Omega
\end{array}
$$

Although one unknown magnetic field is added with this method, the numerical model becomes more convenient since the computation in each iteration step is equivalent to a static 
magnetic field problem with just one source. Moreover, the number of division pieces of fields is not limited. If there are many moving conductors or excitations in the solving domain at the same time, we should correspondingly increase the number of separate fields to express them. And the area with a complex shape could be purposely divided into small, regular ones which are easy to figure.

\section{MOTION ANALYSIS}

In many devices, for instance, the electromagnetic launchers, the motion of the conductor is greatly affected by the magnetic field. The parameter $V$ needs to be solved through the kinetic equations with the results of the magnetic field in each iteration steps. According to Ampere's force law, the force imposed on the conductor by the magnetic field is:

$$
\boldsymbol{F}^{k}=\int \boldsymbol{J}_{e}^{k}(\boldsymbol{x}) \times\left(\nabla \times \boldsymbol{A}^{k}(\boldsymbol{x})\right) d v
$$


where $\boldsymbol{J}_{e}^{k}(\boldsymbol{x})=(1 / \mu) \nabla^{2} \boldsymbol{A}_{e}^{k}(\boldsymbol{x})$.

$\boldsymbol{F}^{k}$ is considered constant during $\Delta t$. So we get:

$\boldsymbol{V}^{k+1}=\boldsymbol{V}^{k}+\boldsymbol{F}^{k} \Delta t / m$

$\operatorname{Dis}^{k}=0.5\left(\boldsymbol{V}^{k}+\boldsymbol{V}^{k+1}\right) \Delta t$

where $m$ is the mass of conductor and Dis $^{k}$ is its moving displacement during $\Delta t$. Sometimes the induction caused by the eddy current magnetic field to the excitation circuit can not be ignored. The inducted electric potential could be written as:

$$
e^{k}=-\int_{s} \frac{\nabla \times \partial \boldsymbol{A}_{e}^{k}(\boldsymbol{x})}{\partial t} \cdot d \boldsymbol{s}
$$

where $d s$ is the actual area of the closed circuit. So $J_{s}$ could be solved Combining (13) with the corresponding circuit equations.

The flow of the method is formulated as:

1. Set $k=0$ and the time $t=t^{0}+k \Delta t$, build up the coordinate systems and the RBF numerical model, initialize the parameters 
and compute $a_{s}^{0}$ and $a_{e}^{0}$.

2. Compute $J_{s}{ }^{1}$ with the corresponding circuit equation, and calculate $a_{s}{ }^{1}$ and $a_{e}{ }^{1}$ with (8) and (9).

3. Compute the magnetic field with (7).

4. Compute the motion and the circuit parameter with (10)-(13).

5. If the motion is over, save the data and end the computation. Otherwise, let $k+1$, modify the distance between the coordinate systems and go to Step 2 to continue the iteration.

\section{NUMERICAL EXAMPLE}

To verify the proposed method, we compute the magnetic field in an electromagnetic switch system and simulate the motion process of the switch.

The switch is an aluminium round plate which could move along an insulated shaft in the center. Two hollow cylindershaped excitation coils are winded with the same outer radius on 
the shaft to control the motion of the switch. Fig. 1 shows the cross-section of the model. The millisecond level fast-rising excitation current is created by the discharge of a capacity. Since the upward and downward motions are driven by circuits with the same parameters, only the upward motion process excited by one coil is analyzed.

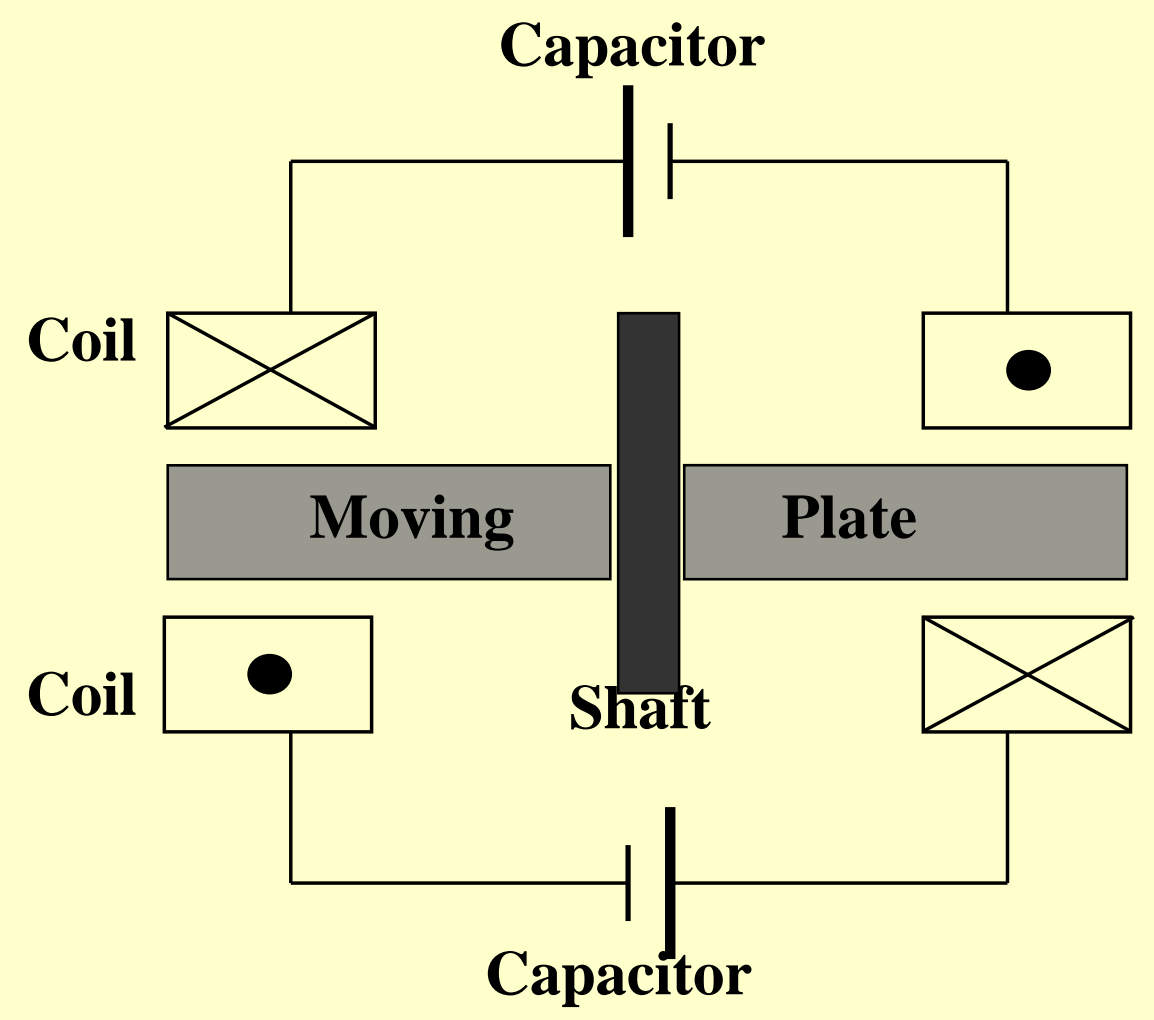

Fig. 1. Cross-section of the electromagnetic switch system.

Because of the rotational symmetry, we only need consider the right side of the cross-section. So the model is equal to a twodimensional domain with a y-direction moving conductive area and a static area with time-variable current below. The simplified model and the sizes are shown in Fig. 2. $A$, and

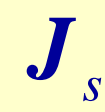


exist only in the z-direction and $A$ satisfies the Dirichlet boundary condition everywhere on $\partial \Omega$. Equation (3) could be written as:

$$
\begin{array}{ll}
\sigma \mu \frac{\partial A_{z}}{\partial t}+\sigma \mu V_{y} \frac{\partial A_{z}}{\partial y}-\nabla^{2} A_{z}=0 & \text { in } \Omega_{e} \\
\nabla^{2} A_{z}=-\mu J_{s} & \text { in } \Omega-\Omega_{e} \\
A_{z}=0 & \text { on } \partial \Omega
\end{array}
$$

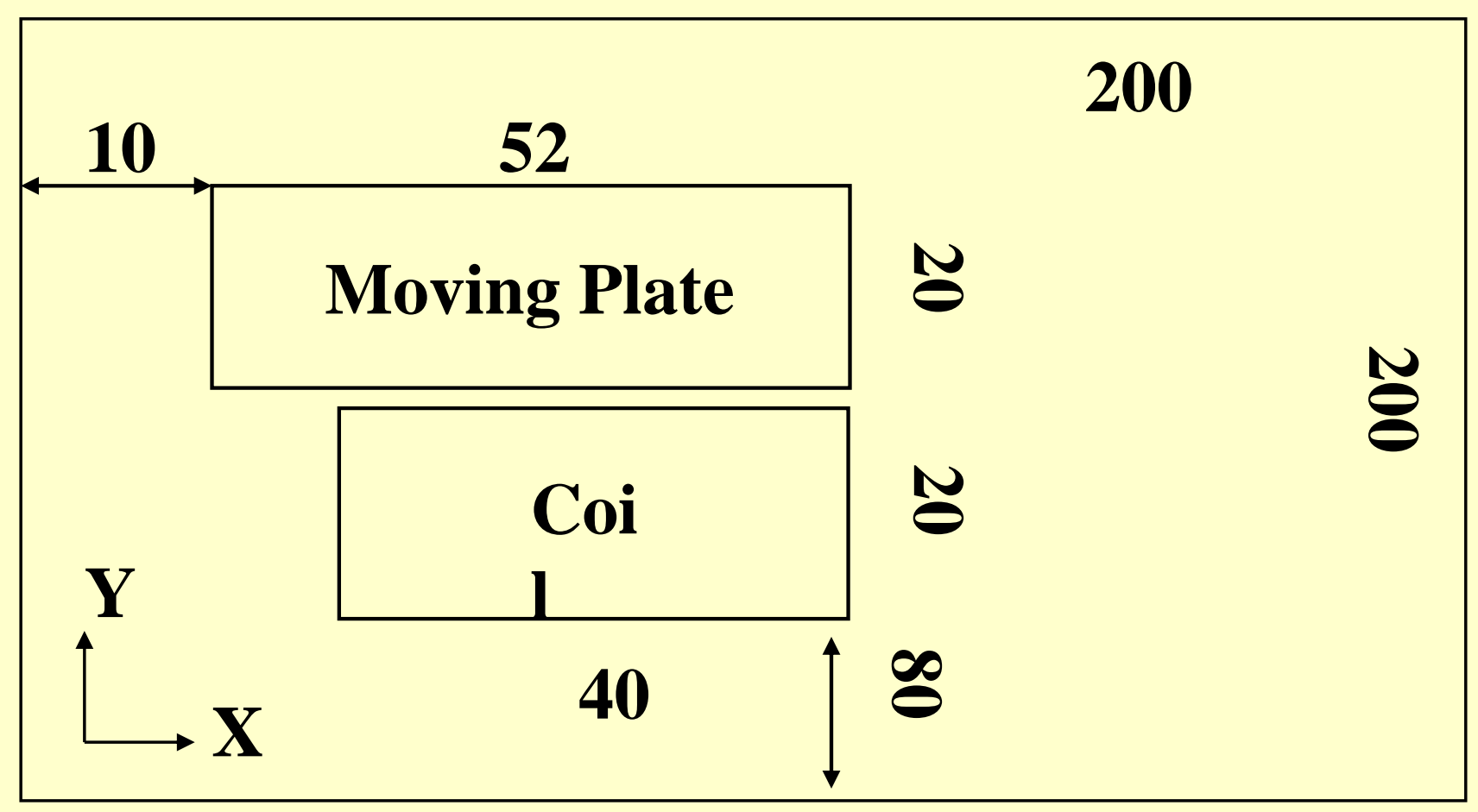

Fig. 2. 2D simplified numerical model of the electromagnetic switch system. (Unit: $\mathrm{mm}$ ) 
The initial distance between the plate and the coil is $3(\mathrm{~mm})$ and the requested distance is $7(\mathrm{~mm})$. The radius of the shaft is $\mathbf{1 0}$ $(\mathrm{mm})$ and there is an initial $3000(\mathrm{~N})$ resisting spring force imposed by the shaft axle on the plate. When the excitation circuit is closed at time $t=0$, the plate, in which the eddy current is generated, is forced to move up by the magnetic field.

The turn number of the coil is 83 and $J_{s}$ is considered homogeneous in the section. The excitation current $I_{s}$ is created by a typical $R L C$ circuit. Because of the eddy current, the inductance needs to be modified in the iteration as:

$$
\begin{aligned}
L^{k+1}= & \rho \frac{d \Phi}{d I_{s}}=\rho \frac{d \Phi_{s}}{d I_{s}}+\rho \frac{d \Phi_{e}}{d I_{s}}=L_{s}+ \\
& \rho\left(\int_{s} \nabla \times\left(\boldsymbol{A}_{e}^{k+1}(\boldsymbol{x})-\boldsymbol{A}_{e}^{k}(\boldsymbol{x})\right) \cdot \boldsymbol{d} \boldsymbol{s}\right) /\left(I_{s}^{k+1}-I_{s}^{k}\right)
\end{aligned}
$$

where $\Phi$ is the magnetic flux and $\rho$ is the interlinkage turns number of the coil, $L_{s}=450(\mu \mathrm{H})$ is the self-inductance of the coil. The capacitance $C=2400(\mu \mathrm{F})$ and the resistance $R=0.06(\Omega)$. 
The initial voltage on the capacitor is $1000(\mathrm{~V})$.

Two coordinate systems are built to compute the magnetic fields. A set of $41 \times 41=1681$ nodes are evenly located in the domain and on the boundary in each of them to form the RBF. According to the model, the initial distance between the coordinate systems is 3 (mm). Here we choose the RBF-Gauss function $\varphi_{j}=\exp \left(-c^{2}\left\|x-x_{j}\right\|^{2}\right)$ for computation.

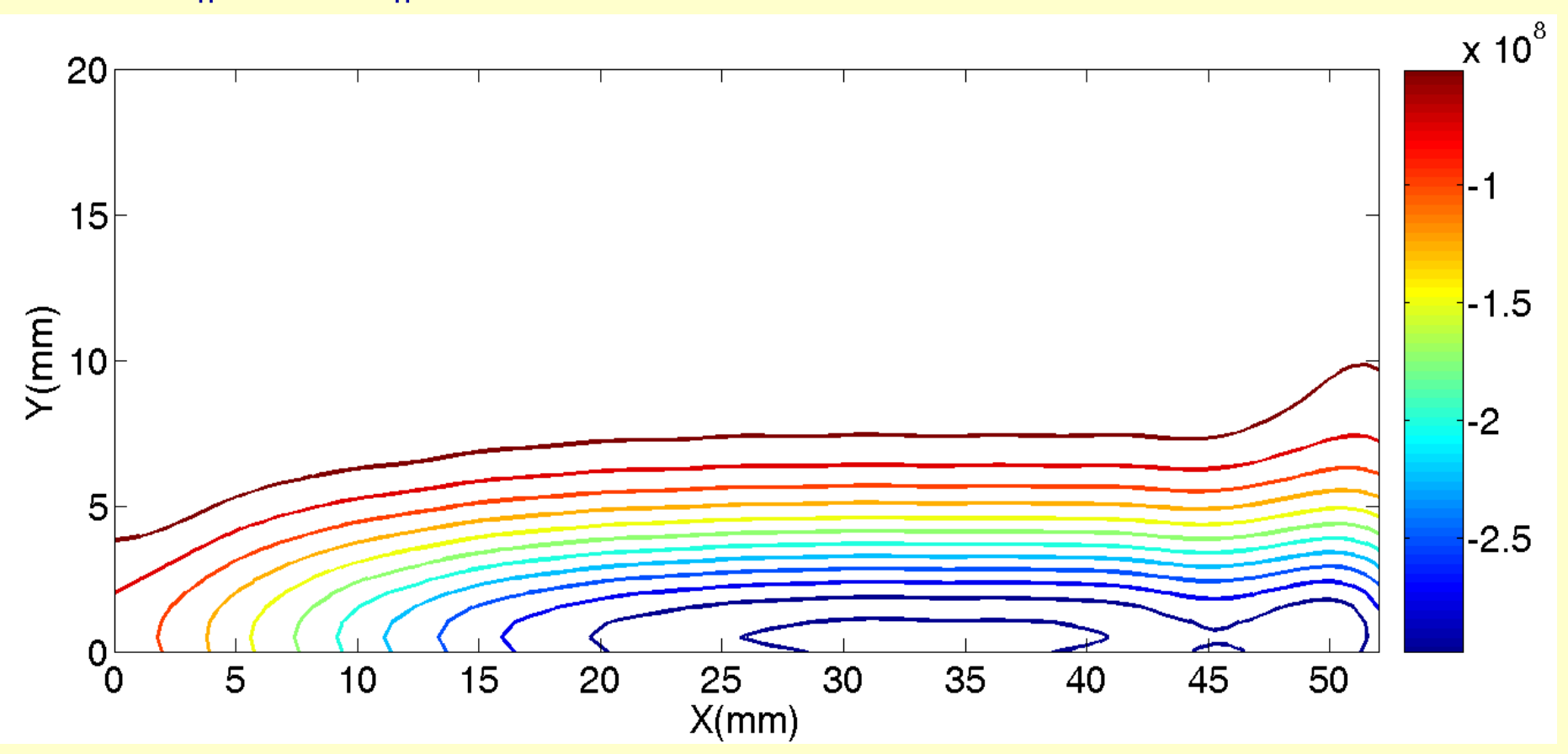

Fig. 3. Isopotential lines of eddy current density in the plate section at $\mathrm{t}=1 \mathrm{~ms}$. 
Fig. 3 shows the isopotential lines of $J_{e}$ in the cross-section of the plate at time $t=1(\mathrm{~ms})$. Furthermore, we choose four observation points from Fig. 3 as: P1(30,0), P2(30,2.5), P3(30,5.0), $P 4(30,7.5)$ and draw their $J_{e}$ time-variation curves in Fig. 4. The curve of $J_{s}$ is also added for compare. From the figure we could clearly see the skin effect and the phase difference.

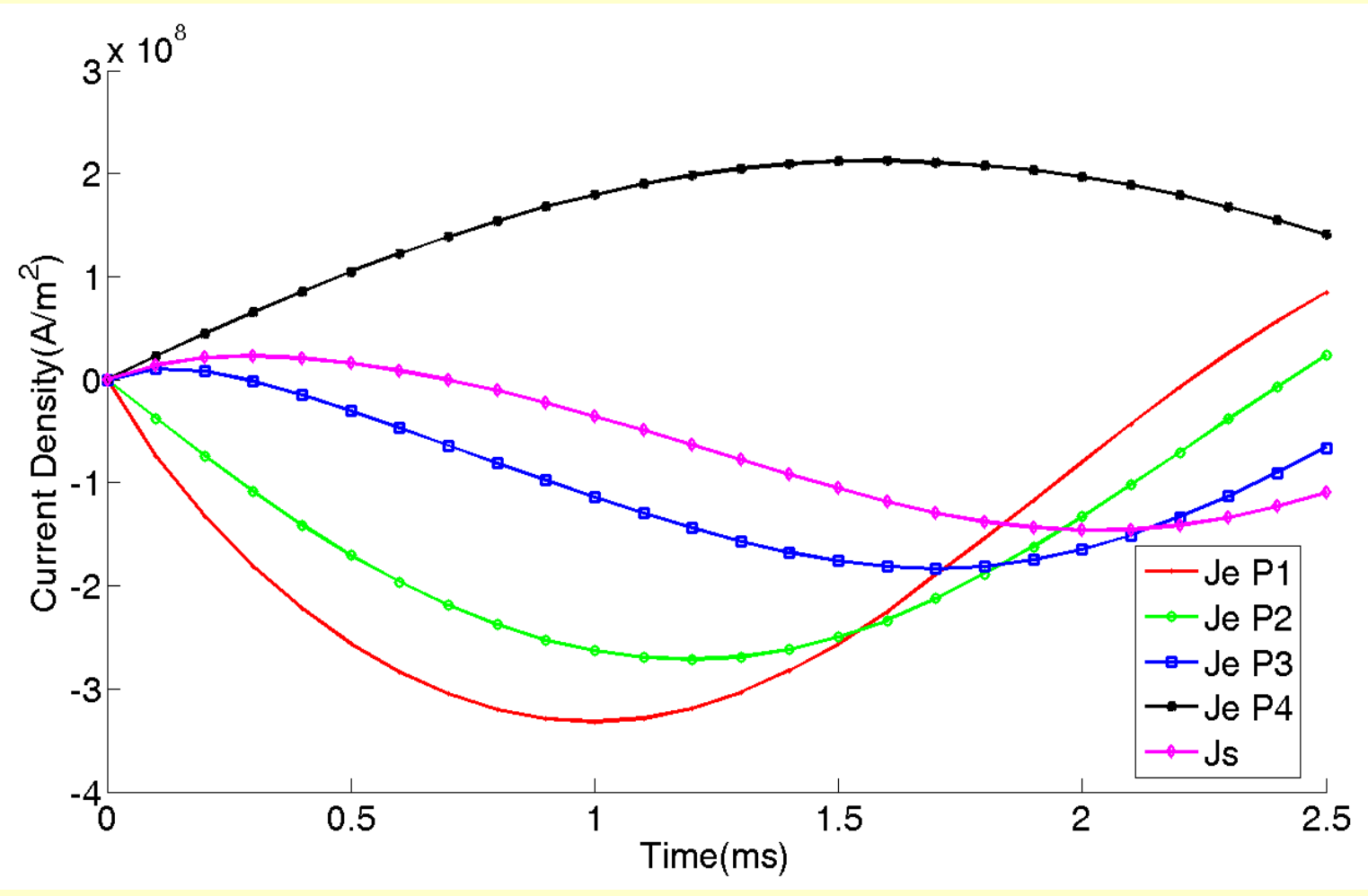

Fig. 4. Time-variation curve of the current densities. 


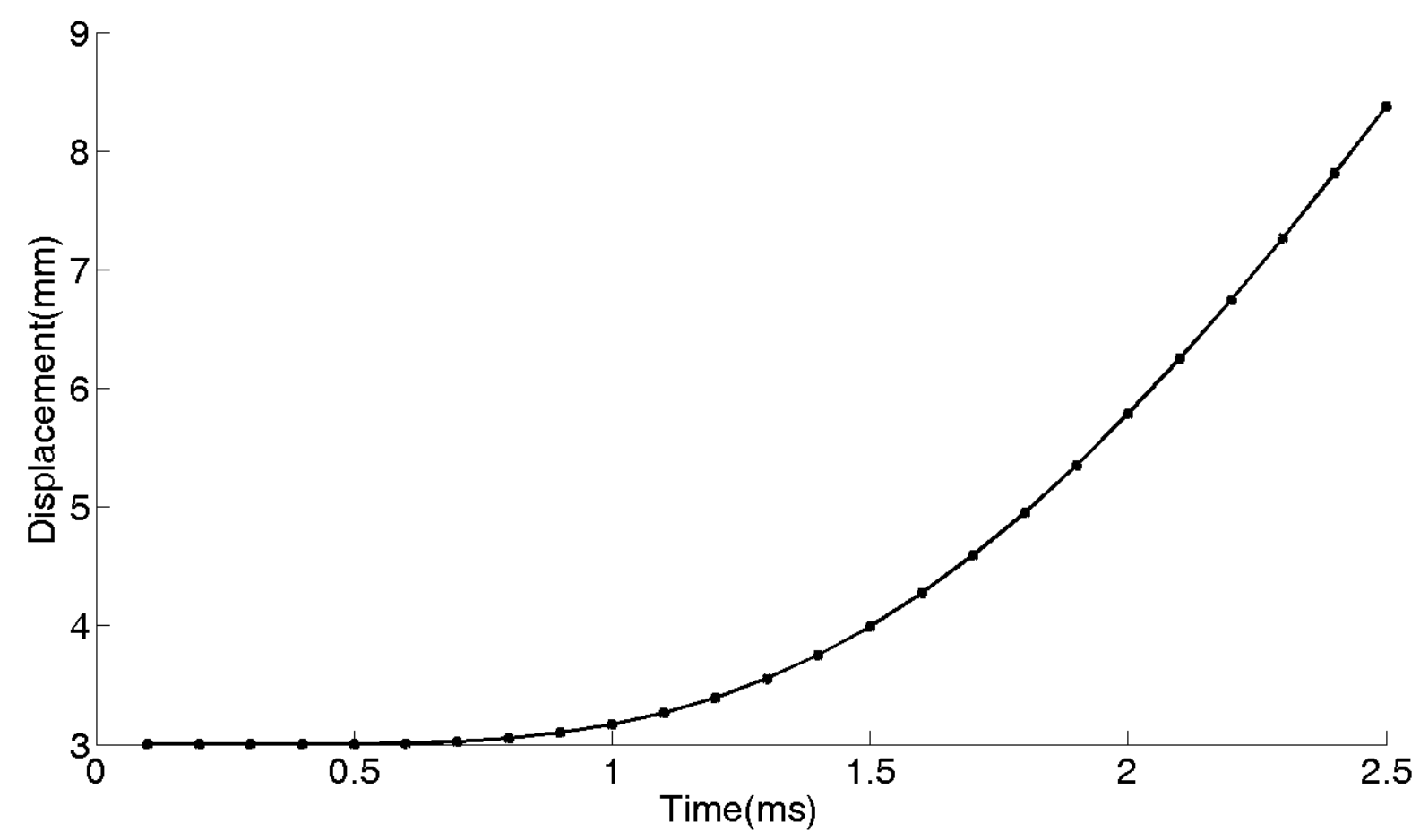

Fig. 5. Variation curve of the moving displacement of the plate

On the basis of the magnetic field, the motion process is simulated. Fig. 5 shows the moving displacement of the plate during the motion. At time $t=0.5(\mathrm{~ms})$, the plate overcame the resisting spring force and began to move. And the plate reached the set position at $t=2.3(\mathrm{~ms})$. The numerical result agrees well with the experiment data. 


\section{CONCLUSION}

A superposition principle based RBF collocation method to compute the moving conductor eddy current problem is presented in this paper. According to the property of RBF, the magnetic field is divided into two separate fields and computed in moving coordinate systems with time domain iteration. Compared with the traditional mesh-based algorithm, this method avoids the model reconfiguration during the computation and could simulate the motion process conveniently. A practical engineering problem is solved to verify the method. The further work is in progress to apply the method to solve other more complex problems such as 3-D problems with asymmetrical shapes. 